\title{
Biological issues with cabozantinib in bone metastatic renal cell carcinoma and castration-resistant prostate cancer
}

\author{
Vincenzo Di Nunno ${ }^{\ddagger}, 1$, Alessia Cimadamore ${ }^{\ddagger 2}$, Matteo Santoni ${ }^{3}$, Marina Scarpelli², \\ Michelangelo Fiorentino ${ }^{4}$, Chiara Ciccarese ${ }^{5}$, Roberto lacovelli5, Liang Cheng ${ }^{6}$, Antonio \\ Lopez-Beltran ${ }^{7}$, Francesco Massari ${ }^{1}$ \& Rodolfo Montironi ${ }^{*, 2}$ \\ ${ }^{1}$ Division of Oncology, S Orsola-Malpighi Hospital, Bologna, Italy \\ 2Section of Pathological Anatomy, Polytechnic University of the Marche Region, School of Medicine, United Hospitals, Ancona, Italy \\ ${ }^{3}$ Oncology Unit, Macerata Hospital, Macerata, Italy \\ ${ }^{4}$ Pathology Department, S Orsola-Malpighi Hospital, Bologna, Italy \\ ${ }^{5}$ Department of Medical Oncology, Azienda Ospedaliera Universitaria Integrata (AOUI), Verona, Italy \\ ${ }^{6}$ Department of Pathology \& Laboratory Medicine, Indiana University School of Medicine, Indianapolis, IN, USA \\ ${ }^{7}$ Department of Surgery, Cordoba University Medical School, Cordoba, Spain \\ *Author for correspondence: r.montironi@univpm.it \\ ${ }^{\ddagger}$ Authors contributed equally
}

\begin{abstract}
"A $\mathrm{A}$ better knowledge of the mechanisms of action as well as of the pathways targeted by such agents appears to be a key point for an improved treatment strategy planning and patients' selection."
\end{abstract}

First draft submitted: 27 February 2018; Accepted for publication: 14 June 2018; Published online: 24 August 2018

Keywords: angiogenesis $\bullet$ bone metastasis $\bullet$ cabozantinib • castration-resistant prostate cancer $\bullet$ MET inhibitor $\bullet$ metastatic RCC • multityrosine kinases inhibitor $\bullet$ osteoblastic activation • renal cell carcinoma $\bullet$ target therapy

The management of metastatic renal cell carcinoma (RCC) has been revolutionized in the last few years by the continuous and steady development of several active drugs. This ongoing process has led to significant improvement in different clinical outcomes and in particular, in patients' survival. A better knowledge of the mechanisms of action as well as of the pathways targeted by such agents appears to be a key point for an improved treatment strategy planning and patients' selection.

\section{Targeted therapies in renal cell carcinoma}

VEGF-targeted therapies have been shown great survival benefit in RCC, but most patients suffer of disease progression after a transient 9-11 month period of clinical benefit. The activation of alternative proangiogenic pathways and the acquisition of invasive and metastatic properties of cancer cells are the main biological mechanisms of evasive resistance to antiangiogenic therapies [1,2].

Mesenchymal-epithelial transition (MET) and AXL expressions are increased in renal cell carcinoma as result of VHL inactivation and are associated with aggressive tumor behavior [3]. Furthermore, the upregulation of MET and AXL are linked to the development of resistance to VEGFR inhibitors in RCC preclinical models [4]. Interestingly, the chronic sunitinib treatment of RCC cell lines activates both AXL and MET and promotes prometastatic behavior and angiogenesis inducing EMT-associated gene expression changes [5]. The therapeutic inactivation of AXL signaling in metastatic clear cell RCC cells has been shown to reverse the invasive and metastatic phenotype in vivo [6]. In vivo studies aimed to assess the effects of drug combination in a human clear cell RCC models provided the rationale for the clinical testing of combined VEGF and HGF/c-met pathway blockade strategy both in firstand second-line setting [7]. 


\section{Multityrosine kinases inhibitor cabozantinib}

Cabozantinib, an oral multityrosine kinases inhibitor that targets MET, VEGFRs and AXL [8], is presently approved for the treatment of patients with metastatic medullary thyroid cancer [9] and is now under evaluation for the treatment of several types of solid cancer, including RCC and prostate cancer [10].

Cabozantinib has improved different clinical outcomes in patients with locally advanced or metastatic RCC when failing to respond to VEGF/VEGFR inhibitors. Recently, Escudier $e$ t al. and Choueiri et al. have described the clinical outcomes achieved in patients with bone metastatic RCC with a clear-cell component treated with cabozantinib versus everolimus in the METEOR trial [11,12].

Although this study is not powered for statistical analysis of subgroups, the outcomes obtained are very interesting in terms of progression-free survival ( 7.4 vs 2.7 months, HR: 0.33 ), objective response rate ( $17 \mathrm{vs} 0 \%$ ) and overall survival (OS; 20.1 vs 10.7 months, HR: 0.45 ). The rate of skeletal-related events was $23 \%$ with cabozantinib and $29 \%$ with everolimus. Furthermore, such outcomes were also seen in patients with both bone and visceral metastases. Considering the poor prognosis and the high rate of complications observed in this subpopulation of metastatic RCC patients, such data have a very important value from the oncologic point of view.

Cabozantinib has also been tested in patients with metastatic castration-resistant prostate cancer (CRPCa) in a Phase III clinical trial (COMET-1), where it failed to demonstrate a clinical benefit in terms of OS [13-15]. However, patients treated with cabozantinib showed a better bone scan response rate ( $42 \%$ with cabozantinib vs $3 \%$ with prednisone), bone biomarkers decrease and circulating tumor cells conversion suggesting an active effect on bone metastases. No distinguish has been made between lytic and condensing bone metastases. According to Som et al. [16], serum 'measurements of bone turnover metabolites during therapy function as clinically informative predictive biomarkers in patients with advanced prostate cancer and skeletal metastases' and could be used as biological surrogate of response for cabozantinib efficacy. The results of the METEOR and COMET-1 trials demonstrate that cabozantinib is an effective treatment in terms of prognosis in bone metastases, an OS benefit seen in metastatic RCC patients with a clear-cell component but not in CRPCa.

\section{Biological issues related to treatment with cabozantinib}

This different benefit in terms of OS in RCC versus CRPCa could be partially explained by the pathways targeted by this drug. One of the most important target of cabozantinib is MET (or HGFR) which plays a critical role in bone metastases development. The $c-M E T$ oncogene "encodes the receptor for the HGF/scatter factor, a cytokine that stimulates the invasive growth of normal and neoplastic cells" [17]. "The MET/HGF receptor is expressed by epithelial cells and its ligand by cells of mesenchymal origin" [17]. Through the MET/HGF receptor, the CD14 ${ }^{+}$ monocytes are differentiated into mature osteoclasts (Figure 1A). Furthermore, MET activation could be triggered by osteoblasts resulting in circulating cells (including tumor cells) migration from blood to bone. Therefore, MET seems to assume a key role since the early phases of metastatic process where its upregulation results in a more aggressive disease and poor prognosis [18,19].

It is possible that the MET pathway could be a key step in bone invasion, shared by different tumors types, including RCC, prostate cancer as well as thyroid and head and neck carcinoma. MET expression could be a 'prerogative' of specific clones of tumor cells that are able to develop distant metastases. This is supported by the fact that there are differences in MET expression from primary tumor and distant metastasis in different tumor types, including head and neck cancer [20]. All this gives support to the clinical efficacy of a MET inhibition in bone metastases.

Why cabozantinib improved OS in patients with metastatic RCC with a clear-cell component while it fails in other tumors? The answer to this question could be searched in the biological activity of this drug as well as in the biological features of RCC. Indeed, other than MET inhibition, cabozantinib is able to target additional key receptors, including AXL and VEGFR, thus also resulting in tumor angiogenesis inhibition. The coinhibition of MET and AXL leads to reduction in cell migration and metastasis development, thus helping to overcome the acquired resistance to VEGFR inhibition [5]. This coinhibition is the explanation why cabozantinib is effective in RCC with bone metastases while other multityrosine kinases inhibitors are not. Indeed, the chronic sunitinib treatment of RCC cell lines promotes prometastatic behavior and angiogenesis activating both AXL and MET [5].

MET hyperexpression is another possible way followed by RCC tumor cells to overcome angiogenesis inhibition. MET could stimulate VEGFA expression through Src activation as well as could lead to an increased angiogenesis by ERK/MAPK/AKT/FAK cascade activation and TSP1 inhibition [21,22]. Considering the close relationship between RCC and angiogenesis it is possible that the favorable outcome achieved in METEOR trial as well as the 
(A)

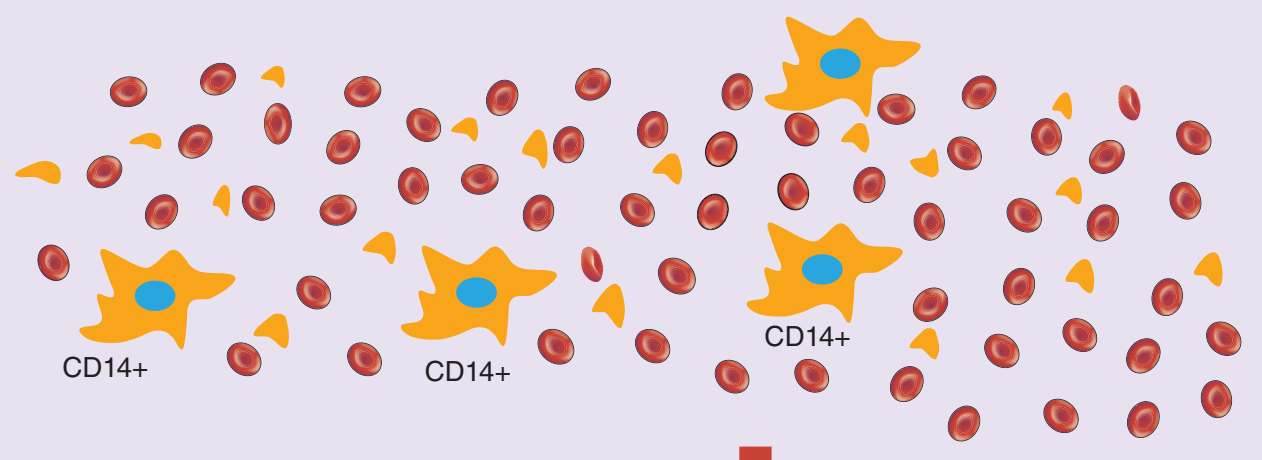

MET/HGF interaction results in RAS-MAPK, RAP1, RAC 1CDC42 and PI3K-AKT pathways activation
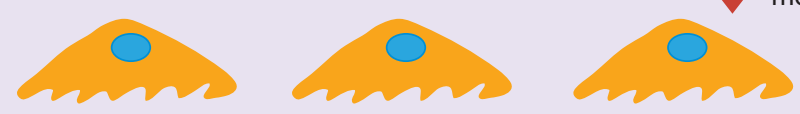

MET/HGF

interaction in CDA14+

monocytes

(B)

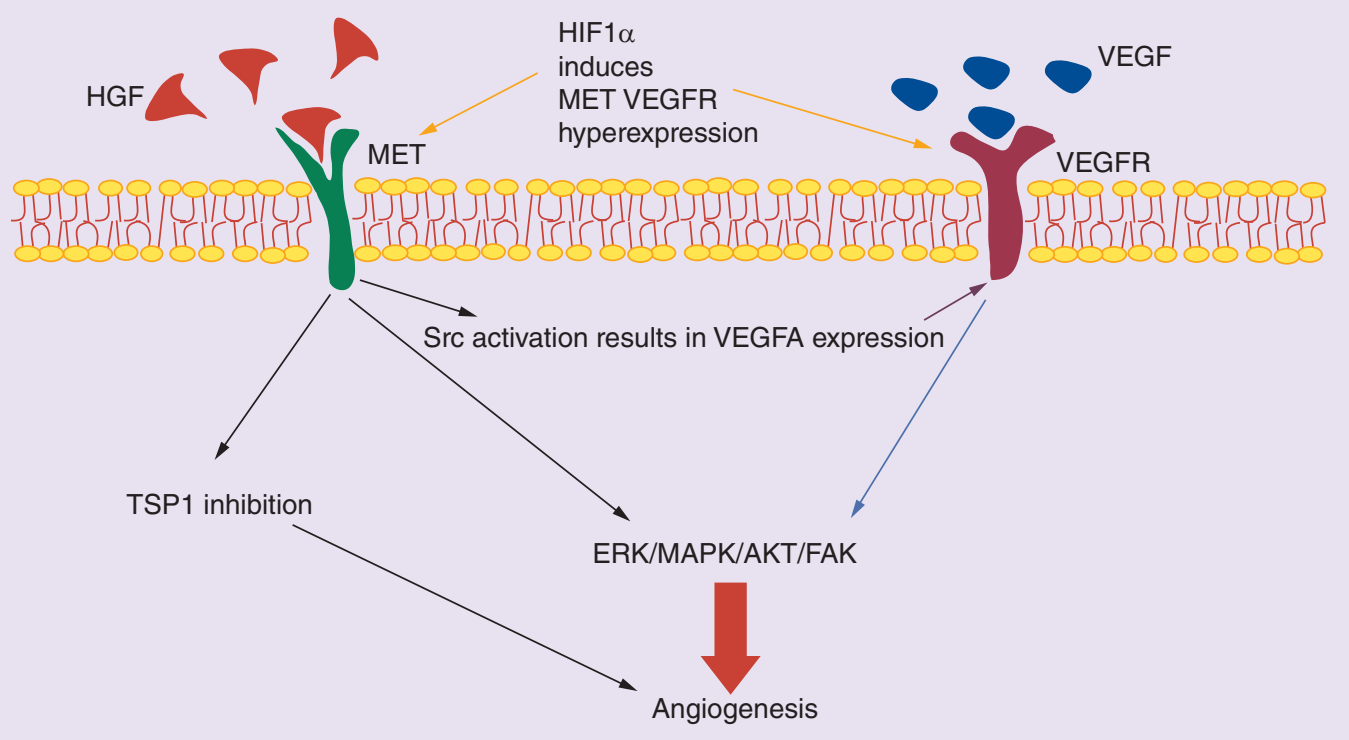

Figure 1. Role of HGF/c-met and VEGF pathways in normal and neoplastic cells. (A) HGF induces differentiation of CD14 ${ }^{+}$monocytes in osteoclasts resulting in osteolysis. Furthermore, HGF produced by osteoblasts stimulates migration of cancer cells from blood to bone marrow space where it supports cancer cells growth. (B) Mesenchymal-epithelial transition and VEGFR activate common signaling intermediates such as ERK, MAPK, AKT and FAK. Mesenchymal-epithelial transition activation can also induce VEGFA expression and angiogenesis through SHCS (SRC homology 2 domain containing proteins). In addition, angiogenesis could be supported through an inhibition HGF/SF mediated of thrombospondin 1 (TSP1) which is a negative regulator of angiogenesis.

MET: Mesenchymal-epithelial transition.

important clinical benefit observed in bone metastatic patients is a consequence of the simultaneous inhibition of different key pathways (Figure 1B).

\section{Patients selection based on target expression}

Another issue to take into consideration is the different expression of MET, AXL and VEGFR among various tumors. This means that patients should be selected according to their cancer-expression of cabozantinib's targets before treatment. Verhoef et al. found that MET protein expression in CRPCa ranged from 1 to 20\% and was observed in the $23 \%$ of hormone resistant prostate cancer patients at palliative trans-urethral resection [23]. MET immunohistochemistry revealed expression in $20-100 \%$ of tumor cells in $18 / 23$ (78\%) prostate cancer 
bone metastasis. Assessing the expressions of cabozantinib's targets at different stages of disease progression is of great value to determine whether subpopulations of prostate cancer patients could be identified for therapeutic stratification.

Varkaris et al. [24], in order to understand mechanisms leading to these responses, conducted parallel investigations on well-defined, representative patient-derived xenografts in conjunction with a Phase II clinical trial as a strategy to provide insights into the unanticipated clinical findings. They demonstrated MET in tumor cells is not a persistent therapeutic target for CRPCa but inhibition of VEGF-R2 and MET in endothelial cells and direct effects on osteoblasts are responsible for cabozantinib-induced tumor inhibition and for the compelling bone scan changes. The primary long-term effect of cabozantinib on tumor growth inhibition/regression was due to targets in the microenvironment and MET signaling is not required for sustained growth of prostate cancer cells in vitro and in vivo. However, vascular heterogeneity represents one source of primary resistance when VEGFR-2 is targeted. Furthermore genetic and pharmacologic inhibition of MET leads to induction of FGFR-1 expression in tumor cells suggesting a potential mechanism of acquired resistance. It also emerged that cabozantinib withdrawal led to rapid, immediate exponential regrowth of all tumors and that continuous treatment is required to inhibit tumor regrowth.

\section{Cabozantinib in combination with other treatment modalities}

"Despite the lack of increase in OS of cabozantinib alone, its combination with other treatment modalities such as abiraterone could potentially optimize treatment outcomes" [25]. In vitro and in vivo experiments showed that cabozantinib can increase the antitumor activity of abiraterone. Enhancing the ability of abiraterone to inhibit AR activity in a cell line-dependent manner. In addition, cabozantinib inhibits the phosphorylation of IGF I receptor, and consequently its activation with downstream activation of MEK1/2 and ERK1/2, that normally results in maintenance of cell survival by antagonizing proteins involved in apoptosis. This mechanism acts as a compensatory survival pathway in response to abiraterone treatment and it is one of the mechanisms for abiraterone resistance in prostate cancer patients.

\section{Conclusion}

The study presented by Escudier et al. and Choueiri et al. [11,12] represents an important contribute for the management of bone metastasis in patients with RCC and a clear-cell component. In particular, the investigation suggests some excellent ideas about the biological role of MET and its interaction with other pathways related to angiogenesis, tumor development and metastatic process. In CRPCa patients, cabozantinib failed to demonstrate a clinical benefit in terms of OS. Answers to this result could be searched in the biological activity of cabozantinib as well as in the different expression of MET, AXL and VEGFR among various tumors. Selecting patients according to their cancer- expression of cabozantinib's targets or combinating cabozantinib with other treatment modalities, such as abiraterone, are suggestions that should be taken into consideration. Further studies exploring MET inhibitors' activity in neoplasms in other organs and tissue will for sure improve our knowledge on the biological role of such pathways and their relationship with treatment with cabozantinib.

Financial \& competing interests disclosure

The authors have no relevant affiliations or financial involvement with any organization or entity with a financial interest in or financial conflict with the subject matter or materials discussed in the manuscript. This includes employment, consultancies, honoraria, stock ownership or options, expert testimony, grants or patents received or pending, or royalties.

No writing assistance was utilized in the production of this manuscript.

\section{References}

Papers of special note have been highlighted as: $\bullet$ of interest; $\bullet \bullet$ of considerable interest

1. Bergers G, Hanahan D. Modes of resistance to antiangiogenic therapy. Nat. Rev. Cancer 8(8), 592-603 (2008).

2. Sennino B, McDonald DM. Controlling escape from angiogenesis inhibitors. Nat. Rev. Cancer 12(10), 699-709 (2012).

3. Gibney GT, Aziz SA, Camp RL et al. C-Met is a prognostic marker and potential therapeutic target in clear cell renal cell carcinoma. Ann. Oncol. 24(2), 343-349 (2013).

- Higher mesenchymal-epithelial transition (MET) expression is detected in all renal cell carcinoma (RCC) subtypes than in the adjacent normal renal tissue and higher MET expression correlated with poor pathologic features and worse disease-specific survival. 
4. Shojaei F, Lee JH, Simmons BH et al. HGF/c-Met acts as an alternative angiogenic pathway in sunitinib-resistant tumors. Cancer Res. 70(24), 10090-10100 (2010).

5. Zhou L, Liu XD, Sun M et al. Targeting MET and AXL overcomes resistance to sunitinib therapy in renal cell carcinoma. Oncogene 35(21), 2687-2697 (2016).

-. The suppression of AXL or MET expression and the inhibition of AXL and MET activation using cabozantinib both impaired chronic sunitinib treatment-induced prometastatic behavior in cell culture and rescued acquired resistance to sunitinib in xenograft models.

6. Rankin EB, Fuh KC, Castellini L et al. Direct regulation of GAS6/AXL signaling by HIF promotes renal metastasis through SRC and MET. Proc. Natl Acad. Sci. USA 111(37), 13373-13378 (2014).

7. Ciamporcero E, Miles KM, Adelaiye R et al. Combination strategy argeting VEGF and HGF/c-met in human renal cell carcinoma models. Mol. Cancer Ther. 14(1), 101-110 (2015).

8. Yakes FM, Chen J, Tan J et al. Cabozantinib (XL184), a novel MET and VEGFR2 inhibitor, simultaneously suppresses metastasis, angiogenesis and tumor growth. Mol. Cancer Ther. 10(12), 2298-2308 (2011).

9. Viola D, Cappagli V, Elisei R. Cabozantinib (XL184) for the treatment of locally advanced or metastatic progressive medullary thyroid cancer. Future Oncol. 9(8), 1083-1092 (2013).

10. Fay AP, Albiges L, Bellmunt J. Current role of cabozantinib in metastatic castration-resistant prostate cancer. Expert Rev. Anticancer Ther. 15(2), 151-156 (2015).

11. Escudier B, Powles T, Motzer RJ et al. Cabozantinib, a new standard of care for patients with advanced renal cell carcinoma and bone metastases? Subgroup analysis of the METEOR trial. J. Clin. Oncol. 36(8), 765-772 (2018).

-. Cabozantinib treatment was associated with improved progression-free survival, overall survival and objective response rate when compared with everolimus treatment in patients with advanced RCC and bone metastases and represents a good treatment option for these patients.

12. Choueiri T, Escudier B, Powles T et al. Cabozantinib versus everolimus in advanced renal-cell carcinoma. N. Engl. J. Med. 373(19), 1814-1823 (2015).

-. Progression-free survival was longer with cabozantinib than with everolimus among patients with RCC that had progressed after VEGFR-targeted therapy.

13. Smith M, De Bono J, Sternberg C et al. Phase III study of cabozantinib in previously treated metastatic castration-resistant prostate cancer: COMET-1. J. Clin. Oncol. 34(25), 3005-3013 (2016)

-. Cabozantinib did not significantly improve overall survival compared with prednisone in patients with mCRPC and progressive disease after docetaxel and abiraterone and/or enzalutamide.

14. Modena A, Massari F, Ciccarese C et al. Targeting Met and VEGFR axis in metastatic castration-resistant prostate cancer: 'game over'? Target Oncol. 11(4), 431-446 (2016).

15. Smith MR, De Bono JS, Sternberg CN et al. Final analysis of COMET-1: cabozantinib (Cabo) versus prednisone (Pred) in metastatic castration-resistant prostate cancer (mCRPC) patients (pts) previously treated with docetaxel (D) and abiraterone (A) and/or enzalutamide (E). J. Clin. Oncol. 33(7 Suppl.), 139-139 (2015).

16. Som A, Tu SM, Liu J et al. Response in bone turnover markers during therapy predicts overall survival in patients with metastatic prostate cancer: analysis of three clinical trials. Br. J. Cancer 107(9), 1547-1553 (2012).

17. Ferracini R, Di Renzo MF, Scotlandi K et al. The Met/HGF receptor is over-expressed in human osteosarcomas and is activated by either a paracrine or an autocrine circuit. Oncogene 10(4), 739-749 (1995).

18. Previdi S, Maroni P, Matteucci E, Broggini M, Bendinelli P, Desiderio MA. Interaction between human-breast cancer metastasis and bone microenvironment through activated hepatocyte growth factor/Met and $\beta$-catenin/Wnt pathways. Eur. J. Cancer 46(9), 1679-1691 (2010).

19. Adamopoulos IE, Xia Z, Lau YS, Athanasou NA. Hepatocyte growth factor can substitute for M-CSF to support osteoclastogenesis. Biochem. Biophys. Res. Commun. 350(2), 478-483 (2006).

20. Di Renzo MF, Olivero M, Martone T et al. Somatic mutations of the MET oncogene are selected during metastatic spread of human HNSC carcinomas. Oncogene 19(12), 1547-1555 (2000).

21. Sulpice E, Ding S, Muscatelli-Groux B et al. Cross-talk between the VEGF-A and HGF signaling pathways in endothelial cells. Biol. Cell 101(9), 525-539 (2009).

22. Zhang YW, Su Y, Volpert OV, Vande Woude GF. Hepatocyte growth factor/scatter factor mediates angiogenesis through positive VEGF and negative thrombospondin 1 regulation. Proc. Natl Acad. Sci. USA 100(22), 12718-12723 (2003).

23. Verhoef EI, Kolijn K, De Herdt MJ et al. MET expression during prostate cancer progression. Oncotarget 7(21), 31029-31036 (2016).

24. Varkaris A, Corn PG, Parikh NU et al. Integrating murine and clinical trials with cabozantinib to understand roles of MET and VEGFR2 as targets for growth inhibition of prostate cancer. Clin. Cancer Res. 22(1), 107-121 (2016). 
-. Demonstrates that MET in tumor cells is not a persistent therapeutic target for metastatic castrate-resistant prostate cancer, but inhibition of VEGFR2 and MET in endothelial cells and direct effects on osteoblasts are responsible for cabozantinib-induced tumor inhibition.

25. Wang X, Huang Y, Christie A et al. Cabozantinib inhibits abiraterone's upregulation of IGFIR phosphorylation and enhances its anti-prostate cancer activity. Clin. Cancer Res. 21(24), 5578-5587 (2015). 